\title{
Existence and nonexistence of positive solutions for a class of Caputo fractional differential equation
}

\author{
Na Wang*, Jin Zhang \\ Department of Applied Mathematics, Shanghai Institute of Technology, Shanghai 201418 China
}

*Corresponding author, e-mail: wangna1621@126.com

Received 14 May 2020

Accepted 8 Nov 2020

\begin{abstract}
This study considers a class of Caputo fractional differential equation. The main results of the existence and nonexistence of positive solutions for a certain problem are obtained by a fixed point theorem. Two examples are demonstrated to illustrate our results.
\end{abstract}

KEYWORDS: Caputo fractional differential equation, integral boundary condition, fixed point theorem, positive solution

MSC2010: 26A33 34B18 37C25 47H10

\section{INTRODUCTION}

Fractional-order differential equation has more advantages compared with integer-order differential equation. This equation is flexible and accurate in describing the changing law of things. Accordingly, fractional-order differential equation is widely used in real life [1-6]. However, some physical meanings of the fractional differential equation are yet to be universally recognized due to the complexity of its initial values; hence, the development of the theory of fractional differential equation is still in its infancy. The fractional differential equation is difficult to study because of the limitation of derivatives in its boundary value problem. However, this equation has become a major topic among many scholars because of its wide practical application value, important theoretical significance, and broad prospects.

Fractional calculus was proposed 300 years ago. The study of fractional differential equations requires the comprehensive application of diverse mathematical knowledge, such as real variable function, complex variable function, integral transformation, functional analysis, and linear algebra. Accordingly, the research of fractional differential equations is difficult and comprehensive.

In addition, fractional differential equations are gradually closely related to other disciplines. The contents of fractional differential equations become rich when they are used in different fields. Many scholars have conducted substantial research on fractional differential equations and their applica- tions, and have obtained good results. Ref. [7] considered a class of four-point fractional boundary value problem of the form

$$
\left\{\begin{array}{l}
D_{0+}^{\alpha} u(x)+\lambda f(x, u(x))=0, \quad x \in(0,1), \\
u^{\prime}(0)-\mu_{1} u(\xi)=0, \quad u^{\prime}(1)-\mu_{2} u(\eta)=0
\end{array}\right.
$$

where $1<\alpha \leqslant 2,0 \leqslant \xi \leqslant \eta \leqslant 1,0 \leqslant \mu_{1}, \mu_{2} \leqslant 1$, $\lambda>0$ are parameters, and $f(x, u):[0,1] \times \mathbb{R}^{+} \rightarrow$ $\mathbb{R}^{+}$is continuous and increasing in $x$ for each $x \in$ $[0,1]$. The authors established the existence and uniqueness of positive solutions for this problem by using the fixed point theorems of concave operators in partial ordering Banach spaces. Ref. [8] studied the boundary value problem of nonlinear fractional differential equations

$$
\left\{\begin{array}{l}
{ }^{C} D^{\alpha} u(x)+f(x, u(x))=0, \quad x \in(0,1) \\
u(0)=u^{\prime}(0)=\cdots=u^{(k-1)}(0) \\
=u^{(k+1)}(0)=\cdots=u^{(n)}(0)=0 \\
u(1)=\lambda \int_{0}^{1} u(t) \mathrm{d} t
\end{array}\right.
$$

where $n<\alpha<n+1, n \geqslant 2,0<k \leqslant n-1, n, k \in \mathbb{N}$, $0<\lambda<k+1,{ }^{C} D^{\alpha}$ is an $\alpha$ order Caputo fractional derivative. The author obtained the existence condition of the positive solution for this equation by using the cone compression and expansion fixed point theorem of a strict set contraction operator.

In Ref. [9], the numerical solutions of the Volterra integro-differential equations are discussed with respect to the initial conditions of necessity by developing the reproducing kernel algorithm 
within the Atangana-Baleanu fractional operator. The solution methodology involves the use of couple Hilbert spaces for the range and domain space. In Ref. [10], Lie symmetry analysis and invariant subspace methods of differential equations play an important role in the study of fractional partial differential equations. The former method helps derive point symmetries, symmetry algebra, and admissible exact solution. Meanwhile, the later one determines an admissible invariant subspace to derive the exact solution of fractional partial differential equations. The results of the existence of fractional differential equations in application are available in the literature. Readers should refer to [11-14].

At present, the main types of fractional derivatives are Caputo type, Riesz type, Riemann-Liouville type, and other fractional derivatives. The Caputo and Riemann-Liouville types of fractional order are widely used among these types because of their broad application background and relatively simple calculation. The research on fractional differential equations keeps increasing every year. However, few studies have been carried out on the existence of positive solutions of Caputo fractional differential equations with complex boundary conditions. This phenomenon is due to two factors. First, a cone and an operator are relatively difficult to construct in the boundary value problem of Caputo fractional differential equation. Second, the proof of inequalities suitable for operator stretching and compression, and the result of the operator's influence on the existence of positive solutions during stretching or compression are difficult to obtain. On the basis of these studies, we will examine the following Caputo fractional differential equation:

$$
\left\{\begin{array}{l}
{ }^{C} D^{\alpha} v(x)+\varphi(x, v(x))=0, \quad 0 \leqslant x \leqslant 1 \\
v(0)=\cdots=v^{(n-1)}(0)=v^{(n+1)}(0)=0, \\
p v(1)+q v^{\prime}(1)=\mu \int_{0}^{\xi} v(t) \mathrm{d} t
\end{array}\right.
$$

where $n+1<\alpha<n+2,0<\mu<n+1, n \in \mathbb{N}, p \geqslant 0$, $q \geqslant 0, p+q \neq 0,0 \leqslant \xi \leqslant 1,0 \leqslant \mu \xi^{\alpha}<p \alpha+\alpha(\alpha-1) q$, $\varphi(x, v):[0,1] \times[0, \infty) \rightarrow[0, \infty)$ is continuous, and ${ }^{C} D^{\alpha}$ is an $\alpha$ order Caputo fractional derivative.

This study aims to obtain the existence and nonexistence of positive solutions for (3). Our main purpose is to prove the existence and nonexistence of solutions for (3) by using a fixed point theorem. Moreover, we illustrate two examples to verify our results.

\section{GREEN FUNCTION}

\section{Construction of the Green function}

Lemma 1 ([15]) Let $\alpha>0$. Then, fractional derivative ${ }^{C} D^{\alpha} v(x)=0$ has a unique solution:

$$
v(x)=\sum_{i=0}^{[\alpha]} \frac{v^{j}(0)}{i !} x^{i}
$$

Lemma 2 ([15]) Let $\alpha>0$. Then,

$$
I^{\alpha C} D^{\alpha} v(x)=v(x)-\sum_{i=0}^{[\alpha]} \frac{v^{j}(0)}{i !} x^{i} .
$$

Lemma 3 Let $\varphi(x) \in C[0,1], n+1<\alpha<n+2, n \in$ $\mathbb{N}, p, q \geqslant 0, p+q \neq, 0<\mu<n+1$, and $0 \leqslant \xi \leqslant 1$. Then, the Caputo-type fractional differential equation

$$
\left\{\begin{array}{l}
{ }^{C} D^{\alpha} v(x)+h(x)=0, \quad 0 \leqslant x \leqslant 1, \\
v(0)=\cdots=v^{(n-1)}(0)=v^{(n+1)}(0)=0, \\
p v(1)+q v^{\prime}(1)=\mu \int_{0}^{\xi} v(t) \mathrm{d} t
\end{array}\right.
$$

has a unique solution $v(x)=\int_{0}^{1} G(x, t) h(t) \mathrm{d} t$, where

$$
\begin{aligned}
& G(x, t)= \\
& \left\{\begin{array}{l}
\frac{\psi(0)(x-t)^{\alpha-1}+(n+1) p x^{n}(1-t)^{\alpha-1}}{\psi(0) \Gamma(\alpha)} \\
+\frac{\alpha(\alpha-1)(n+1) q x^{n}(1-t)^{\alpha-2}-(n+1) \mu x^{n}(\xi-t)^{\alpha}}{\psi(0) \alpha \Gamma(\alpha)}, 0 \leqslant t \leqslant x \leqslant \xi \leqslant 1, \\
\frac{\psi(0)(x-t)^{\alpha-1}+(n+1) p x^{n}(1-t)^{\alpha-1}}{\psi(0) \Gamma(\alpha)} \\
+\frac{(\alpha-1)(n+1) q x^{n}(1-t)^{\alpha-2}}{\psi(0) \Gamma(\alpha)}, 0 \leqslant \xi \leqslant t \leqslant x \leqslant 1, \\
\frac{(n+1) p x^{n}(1-t)^{\alpha-1}+(\alpha-1)(n+1) q x^{n}(1-t)^{\alpha-2}}{\psi(0) \Gamma(\alpha)} \\
\frac{\alpha(n+1) p x^{n}(1-t)^{\alpha-1}+\alpha(\alpha-1)(n+1) q x^{n}(1-t)^{\alpha-2}}{\psi(0) \alpha \Gamma(\alpha)}, 0 \leqslant x \leqslant t \leqslant \xi \leqslant 1, \\
\frac{(n+1) p x^{n}(1-t)^{\alpha-1}+(\alpha-1)(n+1) q x^{n}(1-t)^{\alpha-2}}{\psi(0) \Gamma(\alpha)}, 0 \leqslant \xi \leqslant x \leqslant t \leqslant 1,
\end{array}\right.
\end{aligned}
$$

and $\psi(t)=(n+1) p+n(n+1) q-\mu \xi^{n+1}(1-t)$.

Proof: According to Lemma 2, the first equation of (4) is equivalent to

$$
v(x)=-\frac{1}{\Gamma(\alpha)} \int_{0}^{x}(x-t)^{\alpha-1} h(t) \mathrm{d} t+\sum_{j=0}^{n+1} \frac{v^{j}(0)}{j !} x^{j} .
$$

Together with the second condition of (4), we have

$$
v(x)=-\frac{1}{\Gamma(\alpha)} \int_{0}^{x}(x-t)^{\alpha-1} h(t) \mathrm{d} t+\frac{v^{n}(0)}{n !} x^{n} .
$$


Setting $v^{n}(0) / n !=K$, yields

$$
\begin{aligned}
& v(1)=-\frac{1}{\Gamma(\alpha)} \int_{0}^{1}(1-t)^{\alpha-1} h(t) \mathrm{d} t+K \\
& v^{\prime}(1)=-\frac{1}{\Gamma(\alpha)} \int_{0}^{1}(\alpha-1)(1-t)^{\alpha-2} h(t) \mathrm{d} t+n K
\end{aligned}
$$

and

$$
\int_{0}^{\xi} v(x) d x=-\frac{1}{\Gamma(\alpha)} \int_{0}^{\xi} \frac{(\xi-t)^{\alpha}}{\alpha} h(t) \mathrm{d} t+\frac{\xi^{n+1}}{n+1} K .
$$

From (8)-(10) and the third condition of (4), it yields

$$
\begin{aligned}
K= & \frac{(n+1) p}{\psi(0) \Gamma(\alpha)} \int_{0}^{1}(1-t)^{\alpha-1} h(t) \mathrm{d} t \\
& +\frac{(n+1)(\alpha-1) q}{\psi(0) \Gamma(\alpha)} \int_{0}^{1}(1-t)^{\alpha-2} h(t) \mathrm{d} t \\
& -\frac{(n+1) \mu}{\psi(0) \alpha \Gamma(\alpha)} \int_{0}^{\xi}(\xi-t)^{\alpha} h(t) \mathrm{d} t .
\end{aligned}
$$

Substituting (11) into (7) yields,

$$
\begin{aligned}
v(x)= & \frac{1}{\Gamma(\alpha)} \int_{0}^{x}(x-t)^{\alpha-1} h(t) \mathrm{d} t \\
& +\frac{(n+1) p x^{n}}{\psi(0) \Gamma(\alpha)} \int_{0}^{1}(1-t)^{\alpha-1} h(t) \mathrm{d} t \\
& +\frac{(n+1)(\alpha-1) q x^{n}}{\psi(0) \Gamma(\alpha)} \int_{0}^{1}(1-t)^{\alpha-2} h(t) \mathrm{d} t \\
& -\frac{(n+1) \mu x^{n}}{\psi(0) \alpha \Gamma(\alpha)} \int_{0}^{\xi}(\xi-t)^{\alpha} h(t) \mathrm{d} t .
\end{aligned}
$$

When $0 \leqslant x<\xi \leqslant 1$, we have

$$
\begin{aligned}
& v(x)=\frac{1}{\Gamma(\alpha)} \int_{0}^{x}(x-t)^{\alpha-1} h(t) \mathrm{d} t+\frac{(n+1) p x^{n}}{\psi(0) \Gamma(\alpha)} \times \\
& {\left[\int_{0}^{x}(1-t)^{\alpha-1} h(t) \mathrm{d} t+\int_{x}^{\xi}(1-t)^{\alpha-1} h(t) \mathrm{d} t+\int_{\xi}^{1}(1-t)^{\alpha-1} h(t) \mathrm{d} t\right]} \\
& +\frac{(n+1)(\alpha-1) q x^{n}}{\psi(0) \Gamma(\alpha)}\left[\int_{0}^{x}(1-t)^{\alpha-2} h(t) \mathrm{d} t\right. \\
& \left.+\int_{x}^{\xi}(1-t)^{\alpha-2} h(t) \mathrm{d} t+\int_{\xi}^{1}(1-t)^{\alpha-2} h(t) \mathrm{d} t\right] \\
& -\frac{(n+1) \mu x^{n}}{\psi(0) \alpha \Gamma(\alpha)}\left[\int_{0}^{x}(\xi-t)^{\alpha} h(t) \mathrm{d} t+\int_{x}^{\xi}(\xi-t)^{\alpha} h(t) \mathrm{d} t\right]
\end{aligned}
$$

$$
\begin{aligned}
= & \int_{0}^{x} \frac{\psi(0)(x-t)^{\alpha-1}+(n+1) p x^{n}(1-t)^{\alpha-1}}{\psi(0) \Gamma(\alpha)} h(t) \mathrm{d} t \\
& +\int_{0}^{x} \frac{\alpha(\alpha-1)(n+1) q x^{n}(1-t)^{\alpha-2}-(n+1) \mu x^{n}(\xi-t)^{\alpha}}{\psi(0) \alpha \Gamma(\alpha)} h(t) \mathrm{d} t \\
& +\int_{x}^{\xi} \frac{(n+1) p x^{n}(1-t)^{\alpha-1}+(\alpha-1)(n+1) q x^{n}(1-t)^{\alpha-2}}{\psi(0) \Gamma(\alpha)} h(t) \mathrm{d} t \\
& -\int_{x}^{\xi} \frac{(n+1) \mu x^{n}(\xi-t)^{\alpha}}{\psi(0) \alpha \Gamma(\alpha)} h(t) \mathrm{d} t \\
& +\int_{\xi}^{1} \frac{(n+1) p x^{n}(1-t)^{\alpha-1}+(\alpha-1)(n+1) q x^{n}(1-t)^{\alpha-2}}{\psi(0) \Gamma(\alpha)} h(t) \mathrm{d} t .
\end{aligned}
$$

When $0 \leqslant \xi<x \leqslant 1$, we have

$$
\begin{aligned}
& v(x)=\frac{1}{\Gamma(\alpha)}\left[\int_{0}^{\xi}(x-t)^{\alpha-1} h(t) \mathrm{d} t+\int_{\xi}^{x}(x-t)^{\alpha-1} h(t) \mathrm{d} t\right] \\
& +\frac{(n+1) p x^{n}}{\psi(0) \Gamma(\alpha)}\left[\int_{0}^{\xi}(1-t)^{\alpha-1} h(t) \mathrm{d} t+\int_{\xi}^{x}(1-t)^{\alpha-1} h(t) \mathrm{d} t\right. \\
& \left.+\int_{x}^{1}(1-t)^{\alpha-1} h(t) \mathrm{d} t\right]+\frac{(n+1)(\alpha-1) q x^{n}}{\psi(0) \Gamma(\alpha)} \times \\
& \quad\left[\int_{0}^{\xi}(1-t)^{\alpha-2} h(t) \mathrm{d} t+\int_{\xi}^{x}(1-t)^{\alpha-2} h(t) \mathrm{d} t+\int_{x}^{1}(1-t)^{\alpha-2} h(t) \mathrm{d} t\right] \\
& \quad \frac{(n+1) \mu x^{n}}{\psi(0) \alpha \Gamma(\alpha)}\left[\int_{0}^{\xi}(\xi-t)^{\alpha} h(t) \mathrm{d} t+\int_{\xi}^{x}(\xi-t)^{\alpha} h(t) \mathrm{d} t\right] \\
& =\int_{0}^{\xi} \frac{\psi(0)(x-t)^{\alpha-1}+(n+1) p x^{n}(1-t)^{\alpha-1}}{\psi(0) \Gamma(\alpha)} h(t) \mathrm{d} t \\
& \quad+\int_{0}^{\xi} \frac{\alpha(\alpha-1)(n+1) q x^{n}(1-t)^{\alpha-2}-(n+1) \mu x^{n}(\xi-t)^{\alpha}}{\psi(0) \alpha \Gamma(\alpha)} h(t) \mathrm{d} t \\
& \quad+\int_{\xi}^{x} \frac{\psi(0)(x-t)^{\alpha-1}+(n+1) p x^{n}(1-t)^{\alpha-1}}{\psi(0) \Gamma(\alpha)} h(t) \mathrm{d} t \\
& \quad+\int_{\xi}^{x} \frac{(\alpha-1)(n+1) q x^{n}(1-t)^{\alpha-2}}{\psi(0) \Gamma(\alpha)} h(t) \mathrm{d} t \\
& +\int_{1}^{x} \frac{\alpha(n+1) p x^{n}(1-t)^{\alpha-1}+\alpha(\alpha-1)(n+1) q x^{n}(1-t)^{\alpha-2}}{\psi(0) \alpha \Gamma(\alpha)} h(t) \mathrm{d} t .
\end{aligned}
$$

Thus, Lemma 3 is proved.

\section{Properties of the Green function}

Lemma 4 The Green function $G(x, t)$ defined by formula (5) has the following properties:

$$
\begin{aligned}
& \text { (i) } G(x, t) \geqslant \frac{\left(\alpha^{2}-\alpha\right) q}{\psi(0) \Gamma(\alpha)} x^{n}(1-t)^{\alpha-2} t \\
& +\frac{(n+1) p+n(n+1) q-\mu \xi^{n+1}(1-t)}{\psi(0) \Gamma(\alpha)} x^{n}(1-t)^{\alpha-2}
\end{aligned}
$$


(ii) $G(x, t) \leqslant t(1-t)^{\alpha-1}\left(\frac{n}{\Gamma(\alpha)}+\frac{4 \mu \xi^{\alpha-1}}{\psi(0) \Gamma(\alpha)}\right)$

$$
+\frac{\left(\alpha^{2}-\alpha\right) q}{\psi(0) \Gamma(\alpha)}(1-t)^{\alpha-2} t ;
$$

(iii) $\psi(t)>0$ and $\psi(t)$ is an incremental function on $[0,1]$;

(iv) $G(x, t)>0, \forall x \in(0,1)$.

Proof: When $0 \leqslant t \leqslant x \leqslant \xi \leqslant 1$, we obtain the following expression:

$$
\begin{aligned}
& G(x, t)=\frac{1}{\psi(0) \alpha \Gamma(\alpha)}\left[\alpha \psi(0)(x-t)^{\alpha-1}\right. \\
& +\alpha(n+1) p x^{n}(1-t)^{\alpha-1}+\left(\alpha^{2}-\alpha\right)(n+1) q x^{n}(1-t)^{\alpha-1} \\
& +\left(\alpha^{2}-\alpha\right)(n+1) q x^{n}(1-t)^{\alpha-2}-\left(\alpha^{2}-\alpha\right)(n+1) q x^{n}(1-t)^{\alpha-1} \\
& \left.-(n+1) \mu x^{n} \xi^{\alpha}\left(1-\frac{t}{\xi}\right)^{\alpha}\right] \\
& \geqslant \frac{1}{\psi(0) \alpha \Gamma(\alpha)}\left[\alpha \psi(0)(x-t)^{\alpha-1}\right. \\
& +\alpha(n+1) p x^{n}(1-t)^{\alpha-1}+\left(\alpha^{2}-\alpha\right)(n+1) q x^{n}(1-t)^{\alpha-1} \\
& \left.+\left(\alpha^{2}-\alpha\right)(n+1) q x^{n}(1-t)^{\alpha-2} t-(n+1) \mu x^{n} C^{\alpha}(1-t)^{\alpha}\right] \\
& \geqslant \frac{\left(\alpha^{2}-\alpha\right) q}{\psi(0) \alpha \Gamma(\alpha)} x^{n}(1-t)^{\alpha-2} t-\frac{\psi(t)-\psi(0)}{\psi(0) \alpha \Gamma(\alpha)} x^{n}(1-t)^{\alpha-1} \\
& -\frac{1}{\Gamma(\alpha)}\left[x^{n}(1-t)^{\alpha-1}-(x-t)^{\alpha-1}\right] \\
& \geqslant \frac{\left(\alpha^{2}-\alpha\right) q}{\psi(0) \alpha \Gamma(\alpha)} x^{n}(1-t)^{\alpha-2} t+\frac{\mu t \xi^{n+1}}{\psi(0) \alpha \Gamma(\alpha)} x^{n}(1-t)^{\alpha-1} \\
& -\frac{1}{\Gamma(\alpha)} x^{n}(1-t)^{\alpha-1} \\
& =\frac{\left(\alpha^{2}-\alpha\right) q}{\psi(0) \alpha \Gamma(\alpha)} x^{n}(1-t)^{\alpha-2} t \\
& +\frac{(n+1) p+n(n+1) q-\mu \xi^{n+1}(1-t)}{\psi(0) \Gamma(\alpha)} x^{n}(1-t)^{\alpha-2},
\end{aligned}
$$

and

$$
\begin{aligned}
& G(x, t)=\frac{1}{\psi(0) \alpha \Gamma(\alpha)}\left[\alpha \psi(0)(x-t)^{\alpha-1}\right. \\
& +\alpha(n+1) p x^{n}(1-t)^{\alpha-1}+\left(\alpha^{2}-\alpha\right)(n+1) q x^{n}(1-t)^{\alpha-1} \\
& \left.-(n+1) \mu x^{n}(\xi-t)^{\alpha}+\left(\alpha^{2}-\alpha\right)(n+1) q x^{n}(1-t)^{\alpha-2} t\right] \\
& \leqslant \frac{1}{\psi(0) \alpha \Gamma(\alpha)}\left\{\psi(0) n x^{n+1}(1-t)^{\alpha-2} t(1-x)\right. \\
& \quad+\left(\alpha^{2}-\alpha\right)(n+1) q x^{n}(1-t)^{\alpha-2} t \\
& \left.\quad+\mu \xi^{\alpha} x^{n}(1-t)^{\alpha-1}\left[1-\left(1-\frac{t}{\xi}\right)^{4}\right]\right\} \\
& \leqslant \\
& t(1-t)^{\alpha-1}\left[\frac{n}{\Gamma(\alpha)}+\frac{4 \mu \xi^{\alpha-1}}{\psi(0) \Gamma(\alpha)}\right] \\
& \quad+\frac{\left(\alpha^{2}-\alpha\right) q}{\psi(0) \Gamma(\alpha)}(1-t)^{\alpha-2} t .
\end{aligned}
$$

When $0 \leqslant \xi \leqslant t \leqslant x \leqslant 1$, we obtain the following:

$$
\begin{aligned}
& G(x, t)=\frac{1}{\psi(0) \Gamma(\alpha)}\left[\psi(0)(x-t)^{\alpha-1}+(n+1) p x^{n}(1-t)^{\alpha-1}\right. \\
& \left.\quad+(\alpha-1)(n+1) q x^{n}(1-t)^{\alpha-1}+(\alpha-1)(n+1) q x^{n}(1-t)^{\alpha-2} t\right] \\
& \geqslant \frac{(x-t)^{\alpha-1}}{\Gamma(\alpha)}+\frac{(\alpha-1)(n+1) q x^{n}}{\psi(0) \Gamma(\alpha)}(1-t)^{\alpha-2} t-\frac{\psi(t)}{\psi(0) \Gamma(\alpha)} x^{n}(1-t)^{\alpha-1} \\
& \geqslant \frac{\left(\alpha^{2}-\alpha\right) q}{\psi(0) \Gamma(\alpha)} x^{n}(1-t)^{\alpha-2} t+\frac{\mu \xi^{n+1} t}{\psi(0) \Gamma(\alpha)} x^{n}(1-t)^{\alpha-1}-\frac{1}{\Gamma(\alpha)} x^{n}(1-t)^{\alpha-1} \\
& =\frac{\left(\alpha^{2}-\alpha\right) q}{\psi(0) \Gamma(\alpha)} x^{n}(1-t)^{\alpha-2} t \\
& \quad+\frac{(n+1) p+n(n+1) q-\mu \xi^{n+1}(1-t)}{\psi(0) \Gamma(\alpha)} x^{n}(1-t)^{\alpha-1},
\end{aligned}
$$

and

$$
\begin{aligned}
& G(x, t)=\frac{1}{\psi(0) \Gamma(\alpha)}\left[\psi(0)(x-t)^{\alpha-1}+(n+1) p x^{n}(1-t)^{\alpha-1}\right. \\
& \left.+(\alpha-1)(n+1) q x^{n}(1-t)^{\alpha-1}+(\alpha-1)(n+1) q x^{n}(1-t)^{\alpha-2} t\right] \\
& \leqslant \frac{1}{\psi(0) \Gamma(\alpha)}\left\{\psi(0)\left[(x-t)^{\alpha-1}-x^{n}(1-t)^{\alpha-1}\right]\right. \\
& \left.\quad+\left(\alpha^{2}-\alpha\right) q x^{n}(1-t)^{\alpha-2} t+\mu \xi^{\alpha} x^{n}(1-t)^{\alpha-1}\right\} \\
& \leqslant \frac{n t(1-t)^{\alpha-1}}{\Gamma(\alpha)}+\frac{\left(\alpha^{2}-\alpha\right) q}{\psi(0) \Gamma(\alpha)}(1-t)^{\alpha-2} t+\frac{4 \mu \xi^{\alpha-1}}{\psi(0) \Gamma(\alpha)} t(1-t)^{\alpha-1} \\
& =t(1-t)^{\alpha-1}\left[\frac{n}{\Gamma(\alpha)}+\frac{4 \mu \xi^{\alpha-1}}{\psi(0) \Gamma(\alpha)}\right]+\frac{\left(\alpha^{2}-\alpha\right) q}{\psi(0) \Gamma(\alpha)}(1-t)^{\alpha-2} t .
\end{aligned}
$$

When $0 \leqslant x \leqslant t \leqslant \xi \leqslant 1$, the following expression is achieved:

$$
\begin{aligned}
& G(x, t)=\frac{1}{\psi(0) \alpha \Gamma(\alpha)}\left\{\alpha(n+1) p x^{n}(1-t)^{\alpha-1}\right. \\
& \quad+\left(\alpha^{2}-\alpha\right)(n+1) q x^{n}(1-t)^{\alpha-1}-(n+1) \mu x^{n} \xi^{\alpha}\left(1-\frac{t}{\xi}\right)^{\alpha} \\
& \left.\quad+\left(\alpha^{2}-\alpha\right)(n+1) q x^{n}(1-t)^{\alpha-2} t\right\} \\
& \geqslant \\
& \quad \frac{1}{\psi(0) \alpha \Gamma(\alpha)}\left\{\alpha(n+1) p x^{n}(1-t)^{\alpha-1}\right. \\
& \quad+\left(\alpha^{2}-\alpha\right)(n+1) q x^{n}(1-t)^{\alpha-1}-(n+1) \mu x^{n} \xi^{\alpha}(1-t)^{\alpha} \\
& \left.\quad+\left(\alpha^{2}-\alpha\right)(n+1) q x^{n}(1-t)^{\alpha-2} t\right\} \\
& \geqslant \\
& \geqslant \frac{1}{\psi(0) \Gamma(\alpha)}\left\{\left(\alpha^{2}-\alpha\right)(n+1) q x^{n}(1-t)^{\alpha-2} t-x^{n}(1-t)^{\alpha-1} \psi(t)\right\} \\
& \geqslant \\
& \quad \frac{\left(\alpha^{2}-\alpha\right) q}{\psi(0) \Gamma(\alpha)} x^{n}(1-t)^{\alpha-2} t \\
& \quad+\frac{(n+1) p+n(n+1) q-\mu \xi^{n+1}(1-t)}{\psi(0) \Gamma(\alpha)} x^{n}(1-t)^{\alpha-1},
\end{aligned}
$$


and

$$
\begin{aligned}
& G(x, t)=\frac{1}{\psi(0) \alpha \Gamma(\alpha)}\left\{\alpha(n+1) p x^{n}(1-t)^{\alpha-1}\right. \\
& \quad+\left(\alpha^{2}-\alpha\right)(n+1) q x^{n}(1-t)^{\alpha-1}-(n+1) \mu x^{n}(\xi-t)^{\alpha} \\
& \left.\quad+\left(\alpha^{2}-\alpha\right)(n+1) q x^{n}(1-t)^{\alpha-2} t\right\} \\
& \leqslant \frac{\psi(0)+(n+1) p+n(n+1) q+\psi(0)}{\psi(0) \Gamma(\alpha)} x^{n}(1-t)^{\alpha-1} \\
& \quad+\frac{(\alpha-1)(n+1) q}{\psi(0) \Gamma(\alpha)} x^{n}(1-t)^{\alpha-2} t \\
& \leqslant \\
& t(1-t)^{\alpha-1}\left[\frac{n}{\Gamma(\alpha)}+\frac{4 \mu \xi^{\alpha-1}}{\psi(0) \Gamma(\alpha)}\right]+\frac{\left(\alpha^{2}-\alpha\right) q}{\psi(0) \Gamma(\alpha)}(1-t)^{\alpha-2} t .
\end{aligned}
$$

When $0 \leqslant \xi \leqslant x \leqslant t \leqslant 1$, we have

$$
\begin{aligned}
& G(x, t)=\frac{1}{\psi(0) \Gamma(\alpha)}\left\{[(n+1) p+(\alpha-1)(n+1) q] x^{n}(1-t)^{\alpha-1}\right. \\
& \left.\quad+(\alpha-1)(n+1) q x^{n}(1-t)^{\alpha-2} t\right\} \\
& \geqslant \frac{\left(\alpha^{2}-\alpha\right) q}{\psi(0) \Gamma(\alpha)} x^{n}(1-t)^{\alpha-2} t \\
& \quad+\frac{(n+1) p+n(n+1) q-\mu \xi^{n+1}(1-t)}{\psi(0) \Gamma(\alpha)} x^{n}(1-t)^{\alpha-1},
\end{aligned}
$$

and

$$
\begin{aligned}
& G(x, t)=\frac{1}{\psi(0) \Gamma(\alpha)}\left[(n+1) p x^{n}(1-t)^{\alpha-1}\right. \\
& \left.\quad+(\alpha-1)(n+1) q x^{n}(1-t)^{\alpha-2}\right] \\
& \leqslant \frac{1}{\psi(0) \Gamma(\alpha)}\left\{[(n+1) p+(\alpha-1)(n+1) q] x^{n}(1-t)^{\alpha-1}\right. \\
& \left.\quad+\left(\alpha^{2}-\alpha\right) q x^{n}(1-t)^{\alpha-2} t\right\} \\
& \leqslant \\
& \leqslant(1-t)^{\alpha-1}\left[\frac{n}{\Gamma(\alpha)}+\frac{4 \mu \xi^{\alpha-1}}{\psi(0) \Gamma(\alpha)}\right]+\frac{\left(\alpha^{2}-\alpha\right) q}{\psi(0) \Gamma(\alpha)}(1-t)^{\alpha-2} t .
\end{aligned}
$$

Therefore, properties (i)-(ii) of Lemma 4 are proved. Property (iii) of Lemma 4 is easy to prove by using the expression of $\psi(t)$. Property (iv) of Lemma 4 is obtained by using (i) of Lemma 4. Thus, Lemma 4 is proved.

\section{Lemma 5 (Fixed point theorem of cone [16])}

Let $P$ be a cone in Banach space $E$. Let $\Omega_{1}$ and $\Omega_{2}$ be the two bounded open sets in $E$, and $\theta \in \Omega_{1} \subset \overline{\Omega_{1}} \subset \Omega_{2}, A: P \rightarrow P$ is a fully connected continuation operator. If one of the following conditions is valid:

(i) $\|A x\| \leqslant\|x\|, \forall x \in P \cap \partial \Omega_{1} ;\|A x\| \geqslant\|x\|, \forall x \in$ $P \cap \partial \Omega_{2}$.

(ii) $\|A x\| \geqslant\|x\|, \forall x \in P \cap \partial \Omega_{1} ;\|A x\| \leqslant\|x\|, \forall x \in$ $P \cap \partial \Omega_{2}$.

Then, A must have a fixed point on $P \cap\left(\overline{\Omega_{2}} \backslash \Omega_{1}\right)$.

\section{EXISTENCE OF POSITIVE SOLUTIONS}

We denote the followings for convenience:

$$
\begin{aligned}
\varphi_{0} & =\liminf _{u \rightarrow 0^{+}} \min _{x \in[0,1]} \frac{\varphi(x, u)}{u}, \\
\varphi^{0} & =\limsup _{u \rightarrow 0^{+}} \max _{x \in[0,1]} \frac{\varphi(x, u)}{u}, \\
\varphi_{\infty} & =\liminf _{u \rightarrow \infty} \min _{x \in[0,1]} \frac{\varphi(x, u)}{u}, \\
\varphi^{\infty} & =\limsup _{u \rightarrow \infty} \max _{x \in[0,1]} \frac{\varphi(x, u)}{u}, \\
A & =\max _{\{}\left\{\frac{n}{\Gamma(\alpha)}+\frac{4 \mu \xi^{\alpha-1}}{\psi(0) \Gamma(\alpha)}, \frac{\left(\alpha^{2}-\alpha\right) q}{\psi(0) \Gamma(\alpha)}\right\}, \\
a & =\min _{\bar{A}}\left\{\frac{\left(\alpha^{2}-\alpha\right) q}{\psi(0) \Gamma(\alpha)}, \frac{(n+1) p+n(n+1) q-\mu \xi^{\alpha-1}}{\psi(0) \Gamma(\alpha)}\right\} \\
\bar{B} & =\left(\int_{0}^{1} A t(1-t)^{\alpha-2}(2-t) \mathrm{d} t\right)^{-1}, \\
\tau & =\left(\frac{a^{2}}{A} \int_{0}^{1}\left(\frac{1}{2}\right)^{\alpha-1} t^{\alpha}(1-t)^{\alpha-2}(2-t) \mathrm{d} t\right)^{-1}, \\
\tilde{B} & =\left(\int_{1 / 4}^{3 / 4}\left(\frac{1}{2}\right)^{\alpha-1} a \varphi t(1-t)^{\alpha-2}(2-t) \mathrm{d} t\right)^{-1}, \\
& =\min _{x \in\left[\frac{1}{4}, \frac{3}{4}\right]} \frac{a t^{\alpha-1}}{A}=\frac{a}{A}\left(\frac{1}{4}\right)^{\alpha-1} .
\end{aligned}
$$

The conditions used in this study are as follows:

$\left(\mathrm{C}_{1}\right) \varphi(x, u)$ is continuous function, and $\varphi(x, u):[0,1] \times[0, \infty) \rightarrow[0, \infty)$;

$\left(\mathrm{C}_{2}\right) \varphi_{0}>\bar{B}, \varphi_{\infty}>\tilde{B}$;

$\left(\mathrm{C}_{3}\right) \varphi^{0}>\bar{a}, \varphi^{\infty}>\bar{a}$;

$\left(\mathrm{C}_{4}\right)$ Let $\omega>0,0 \leqslant u \leqslant \omega, 0 \leqslant x \leqslant 1$.

Then $\varphi(x, u) \leqslant \omega \bar{A}$;

$\left(\mathrm{C}_{5}\right)$ Let $\omega>0, \omega \tau \leqslant u \leqslant \omega, \frac{1}{4} \leqslant x \leqslant \frac{3}{4}$.

Then $\varphi(x, u)>u \tilde{B}$.

Remark 1 If we take $\omega>0,0 \leqslant u \leqslant \omega, 0 \leqslant x \leqslant 1$, then $\varphi(x, u) \leqslant u \bar{A}$. Therefore, condition $\left(\mathrm{C}_{4}\right)$ holds.

Remark 2 If we take $\omega>0, \omega \tau \leqslant u \leqslant \omega, \frac{1}{4} \leqslant x \leqslant$ $\frac{3}{4}$, then $\varphi(x, u)>\omega \tilde{B}$. Therefore, condition $\left(\mathrm{C}_{5}\right)$ holds.

Let $E=C[0,1]$ and $\|v\|=\max _{x \in[0,1]}|v(x)|$. Then, $E$ is Banach space. Cone $P$ in $E$ is defined as follows:

$$
P=\left\{v \in E \mid v(x) \geqslant \frac{a x^{\alpha-1}}{A}\|v(x)\|\right\} .
$$

We denote the operator as follows:

$$
T v(x)=\int_{0}^{1} G(x, t) \varphi(t, v(t)) \mathrm{d} t, \quad v \in E .
$$


Lemma 6 If condition $\left(C_{1}\right)$ holds, then $T(P) \subset P, T$ : $P \rightarrow P$, and operator $T$ is completely continuous.

Proof: The proof of is similar to the corresponding proof in Ref. [17] and will be omitted here.

Theorem 1 Suppose that conditions $\left(C_{1}\right),\left(C_{2}\right)$, and $\left(C_{4}\right)$ hold. Then, we have at least two positive solutions $\left(v_{1}\right.$ and $v_{2}$ ) of boundary value problem (3) and $0<\left\|v_{1}\right\|<\omega<\left\|v_{2}\right\|$, where $\|v\|=\sup _{x \in[0,1]}|v(x)|$.

Proof: Accordingly to condition $\varphi_{0}>\bar{B}$ of $\left(\mathrm{C}_{2}\right)$, there exist $\varepsilon>0$ and $0<m_{0}<\omega$ such that

$$
\varphi_{0}-\varepsilon>\bar{B} \text { and } v \bar{B}<V\left(\varphi_{0}-\varepsilon\right)<\varphi(x, v)
$$

for all $x \in[0,1], 0 \leqslant v \leqslant m_{0}$. Let $m \in\left(0, m_{0}\right), \Omega_{m}=$ $\{v \in P \mid\|v\|<m\}$, and then for all $v \in \partial \Omega_{m}$, we have

$$
\frac{a x^{\alpha-1}}{A} m \leqslant v(x) \leqslant m, \quad x \in[0,1] \text {. }
$$

Then

$$
\begin{aligned}
(T v)\left(\frac{1}{2}\right) & =\int_{0}^{1} G\left(\frac{1}{2}, t\right) \varphi(t, v(t)) \mathrm{d} t \\
& \geqslant \int_{0}^{1} a\left(\frac{1}{2}\right)^{\alpha-1} t(1-t)^{\alpha-2}(2-t) \bar{B} v(t) \mathrm{d} t \\
& \geqslant a\left(\frac{1}{2}\right)^{\alpha-1} \bar{B} \int_{0}^{1} \frac{a}{A} t^{\alpha-1} m t(1-t)^{\alpha-2}(2-t) \mathrm{d} t \\
& \geqslant \bar{B} m \int_{0}^{1} \frac{a^{2}}{A}\left(\frac{1}{2}\right)^{\alpha-1} t^{\alpha}(1-t)^{\alpha-2}(2-t) \mathrm{d} t \\
& =m=\|v\| .
\end{aligned}
$$

Specifically $\|v\|<\|T v\|$, for all $v \in \partial \Omega_{m}$.

According to condition $\varphi_{\infty}>\tilde{B}$ of $\left(\mathrm{C}_{2}\right)$, there exist $\varepsilon>0$ and $Z>0$ such that

$$
\varphi_{0}-\varepsilon>\tilde{B} \quad \text { and } \quad v \tilde{B}<V\left(\varphi_{\infty}-\varepsilon\right)<\varphi(x, v)
$$

for all $v \geqslant Z$. We take $M>M_{0}=\max \left\{\frac{Z}{\tau}, \omega\right\}$ and $\Omega_{M}=\{v \in P \mid\|v\|<M\}$. Given $Z \leqslant \tau\|v\| \leqslant v(x)$, for all $v \in \partial \Omega_{M}$ and $x \in\left[\frac{1}{4}, \frac{3}{4}\right]$, we have

$$
\begin{aligned}
(T v)\left(\frac{1}{2}\right) & =\int_{0}^{1} G\left(\frac{1}{2}, t\right) \varphi(t, v(t)) \mathrm{d} t \\
& \geqslant \int_{1 / 4}^{3 / 4} a\left(\frac{1}{2}\right)^{\alpha-1} t(1-t)^{\alpha-2}(2-t) \tilde{B} v(t) \mathrm{d} t \\
& \geqslant \tilde{B} M \int_{1 / 4}^{3 / 4} \tau a\left(\frac{1}{2}\right)^{\alpha-1} t(1-t)^{\alpha-2}(2-t) \mathrm{d} t \\
& =M=\|v\| .
\end{aligned}
$$

Specifically, $\|v\|<\|T v\|$, for all $v \in \partial \Omega_{M}$.

Let $\Omega_{r}=\{v \in P \mid\|v\| \in r\}$. According to $\left(\mathrm{C}_{4}\right)$, for all $v \in \partial \Omega_{r}$ and $x \in[0,1]$, we have $\varphi(x, v) \leqslant \bar{A} \omega$. Then,

$$
\begin{aligned}
(T v)\left(\frac{1}{2}\right) & \leqslant A \int_{0}^{1}\left(t(1-t)^{\alpha-1}+t(1-t)^{\alpha-2}\right) \varphi(t, v(t)) \mathrm{d} t \\
& \leqslant \bar{A} r \int_{0}^{1} A(1-t)^{\alpha-2} t(2-t) \varphi(t, v(t)) \mathrm{d} t \\
& =r=\|v\| .
\end{aligned}
$$

Specifically, $\|T v\|<\|v\|$, for all $v \in \partial \Omega_{r}$. In summary, Theorem 1 is proved from Lemma 5.

Remark 3 Condition $\left(\mathrm{C}_{2}\right)$ can be replaced by $\left(\mathrm{C}_{2}^{*}\right)$. $\left(C_{2}^{*}\right) \varphi_{0}=\infty, \varphi_{\infty}=\infty$.

Theorem 2 Suppose that conditions $\left(C_{1}\right),\left(C_{3}\right)$, and $\left(C_{5}\right)$ hold, then at least two positive solutions $\left(v_{1}\right.$ and $v_{2}$ ) of boundary value problem (3) are present, and $0<\left\|v_{1}\right\|<\omega<\left\|v_{2}\right\|$.

Proof: According to condition $\varphi^{0}<\bar{A}$ of $\left(\mathrm{C}_{3}\right)$, there exist $\varepsilon>0$ and $0<m_{0}<\omega$ such that

$$
\varphi^{0}+\varepsilon<\bar{A} \text { and } \varphi(x, v)<V\left(\varphi^{0}+\varepsilon\right)<v \bar{A}
$$

for all $x \in[0,1], 0 \leqslant v \leqslant m_{0}$.

Let $m \in\left(0, m_{0}\right)$, and $\Omega_{m}=\{v \in P \mid\|v\|<m\}$ for all $v \in \partial \Omega_{m}$. We have

$$
\frac{a x^{\alpha-1}}{A} m \leqslant v(x) \leqslant m, \quad x \in[0,1] .
$$

Then,

$$
\begin{aligned}
(T v)(x) & =\int_{0}^{1} G(x, t) \varphi(t, v(t)) \mathrm{d} t \\
& \leqslant \int_{0}^{1} A t(1-t)^{\alpha-2}(2-t) \bar{A} m \mathrm{~d} t \\
& \leqslant A \bar{A} m \int_{0}^{1} t(1-t)^{\alpha-2}(2-t) \mathrm{d} t \\
& =m=\|v\| .
\end{aligned}
$$

Therefore, $\|v\|>\|T v\|, \forall v \in \partial \Omega_{m}$.

According to condition $\varphi^{\infty}<\bar{A}$ of $\left(\mathrm{C}_{3}\right)$, there exist $\varepsilon>0$ and $Z>0$ such that

$$
\varphi^{\infty}+\varepsilon<\bar{A} \text { and } \varphi(x, v) \leqslant v\left(\varphi^{\infty}+\varepsilon\right)<v \bar{B}
$$


for all $v \geqslant Z$. We take $M>M_{0}=\max \{m+\omega, Z\}$. If $\max _{x \in[0,1]} \varphi(x, v)$ is unbounded on $v \in[0, \infty)$, then

$$
\varphi(x, v) \leqslant \max _{x \in[0,1]} \varphi(x, M), \quad \forall v \in(0, M) .
$$

We obtain the following expression for all $v \in P$ and $\|v\|=M$

$$
\begin{aligned}
\|T v\| & \leqslant A \int_{0}^{1}\left(t(1-t)^{\alpha-1}+t(1-t)^{\alpha-2}\right) \varphi(t, v(t)) \mathrm{d} t \\
& \leqslant \int_{0}^{1} A t(1-t)^{\alpha-2}(2-t) \max _{x \in[0,1]} \varphi(t, M) \mathrm{d} t \\
& \leqslant\left(\varphi^{\infty}+\varepsilon\right) M \int_{0}^{1} A t(1-t)^{\alpha-2}(2-t) \mathrm{d} t \\
& =M=\|v\| .
\end{aligned}
$$

If $\max _{x \in[0,1]} \varphi(x, v)$ is bounded on $v \in[0, \infty)$, then we have $\varphi(x, v) \leqslant N$ for all $v \in[0, \infty)$. Let $M>$ $\omega+\frac{N}{\bar{A}}$, and $\|v\|=M$ for all $v \in P$. Then, we obtain

$$
\begin{aligned}
\|T v\| & \leqslant A \int_{0}^{1}\left(t(1-t)^{\alpha-1}+t(1-t)^{\alpha-2}\right) N \mathrm{~d} t \\
& \leqslant \frac{N}{\bar{A}}<M=\|v\| .
\end{aligned}
$$

Let $\Omega_{M}=\{v \in P \mid\|v\|<M\}$ for all $v \in \partial \Omega_{M}$. Hence, we have $\|T v\|<\|v\|$.

We take $\Omega_{r}=\{v \in P \mid\|v\|<r\}$. According to $\left(\mathrm{C}_{3}\right)$, we have

$$
\tau \omega \leqslant v(x) \leqslant \omega \text { for all } v \in \partial \Omega_{r}, x \in\left[\frac{1}{4}, \frac{3}{4}\right],
$$

and

$$
\varphi(x, v(x))>v \tilde{B}
$$

Then,

$$
\begin{aligned}
\|T v\| & =\int_{0}^{1} G\left(\frac{1}{2}, t\right) \varphi(t, v(t)) \mathrm{d} t \\
& \geqslant \int_{1 / 4}^{3 / 4} a\left(\frac{1}{2}\right)^{\alpha-1} t(1-t)^{\alpha-2}(2-t) \tilde{B} v(t) \mathrm{d} t \\
& \geqslant \tilde{B} r \int_{1 / 4}^{3 / 4} \tau a\left(\frac{1}{2}\right)^{\alpha-1} t(1-t)^{\alpha-2}(2-t) \mathrm{d} t \\
& =r=\|v\| .
\end{aligned}
$$

Specifically $\|T v\|>\|v\|$ for all $v \in \partial \Omega_{r}$. In summary, Theorem 2 is proved from Lemma 5.

Remark 4 Condition $\left(\mathrm{C}_{3}\right)$ can be replaced by $\left(\mathrm{C}_{3}^{*}\right)$. $\left(\mathrm{C}_{3}^{*}\right) \varphi^{0}=0, \varphi^{\infty}=0$.
Remark 5 We only need to construct $\Omega_{3}, \Omega_{4}, \cdots, \Omega_{n}$ in cone $P$ to obtain the existence of the $n$th positive solutions. Accordingly, $\Omega_{3} \subset \Omega_{4} \subset \cdots \subset \Omega_{n}$. Then, the fixed point theorem can be used on $\overline{\Omega_{i}} \backslash \Omega_{i-1},(i=$ $3, \cdots, n)$.

\section{NONEXISTENCE OF A POSITIVE SOLUTION}

The following expression is obtained from properties (i) and (ii) of the Green functions of Lemma 4:

$$
\begin{aligned}
G(x, t) \geqslant & \frac{\left(\alpha^{2}-\alpha\right) q}{\psi(0) \Gamma(\alpha)} x^{n}(1-t)^{\alpha-2} t \\
& +\frac{(n+1) p+n(n+1) q-\mu \xi^{n+1}(1-t)}{\psi(0) \Gamma(\alpha)} x^{n}(1-t)^{\alpha-2} \\
\geqslant & \frac{\alpha^{2}-\alpha}{\psi(0) \Gamma(\alpha)}
\end{aligned}
$$

and

$$
\begin{aligned}
G(x, t) \leqslant & t(1-t)^{\alpha-1}\left[\frac{n}{\Gamma(\alpha)}+\frac{4 \mu \xi^{\alpha-1}}{\psi(0) \Gamma(\alpha)}\right] \\
& +\frac{\left(\alpha^{2}-\alpha\right) q}{\psi(0) \Gamma(\alpha)}(1-t)^{\alpha-2} t \\
\leqslant & \frac{\psi(0) n+4 \mu+\left(\alpha^{2}-\alpha\right) q}{\psi(0) \Gamma(\alpha)} .
\end{aligned}
$$

Theorem 3 Suppose that one of the following conditions holds

(i) $\lim _{v \rightarrow+\infty} \inf _{x \in[0,1]}\left(\frac{\varphi(x, v)}{\|v\|}\right)>\frac{\alpha^{2}-\alpha}{\psi(0) \Gamma(\alpha)}$;

(ii) $\lim _{v \rightarrow+\infty} \sup _{x \in[0,1]}\left(\frac{\varphi(x, v)}{\|v\|}\right)<\frac{\varphi(0) n+4 \mu+\left(\alpha^{2}-\alpha\right) q}{\psi(0) \Gamma(\alpha)}$;

(iii) $\frac{\varphi(x, v)}{\|v\|}>\frac{\alpha^{2}-\alpha}{\psi(0) \Gamma(\alpha)}$;

(iv) $\frac{\varphi(x, v)}{\|v\|}<\frac{\varphi(0) n+4 \mu+\left(\alpha^{2}-\alpha\right) q}{\psi(0) \Gamma(\alpha)}$.

Then, boundary value problem (3) does not have a positive solution.

Proof: We will prove by contradiction. Suppose that boundary value problem (3) has two positive solutions $\left(v_{1}\right.$ and $\left.v_{2}\right)$. From (i), we have

$$
\varphi(x, v)>v_{1},\|v\| \|>v_{2} .
$$

Let $M_{1}^{*}=\min _{0 \leqslant v \leqslant v_{2}} \varphi(x, v)$. Then

$$
\varphi(x, v)>v_{1}\|v\|+M_{1}^{*}, \quad v \in[0, \infty) .
$$

If boundary problem (3) has a positive solution $v$, and $\|v\|=\theta$, then $v$ is a fixed point on mapping $T$. Thus, we obtain the following expression:

$$
\begin{gathered}
\theta=\|T v\|=\max _{x \in[0,1]} \int_{0}^{1} G(x, t) \varphi(x, v) \mathrm{d} t \\
>\frac{\alpha^{2}-\alpha}{\psi(0) \Gamma(\alpha)} \cdot \frac{\psi(0) \Gamma(\alpha)}{\alpha^{2}-\alpha} \cdot\|v\|=\theta .
\end{gathered}
$$


This expression contradicts the hypothesis.

Suppose that boundary value problem (3) has two positive solutions $\left(v_{3}\right.$ and $\left.v_{4}\right)$. From (ii), we have

$$
0<v_{3}<\frac{\psi(0) n+4 \mu+\left(\alpha^{2}-\alpha\right) q}{\psi(0) \Gamma(\alpha)} .
$$

We have

$$
\varphi(x,[v])>v_{3}\|v\|, \quad v>v_{4} .
$$

Let $M_{2}^{*}=\min _{0 \leqslant v \leqslant v_{4}} \varphi(x,[v])$. Then,

$$
\varphi(x,[v])<v_{3}\|v\|+M_{2}^{*}, \quad v \in[0, \infty) .
$$

If boundary problem (3) has a positive solution $v$, and $\|v\|=\theta$, then $v$ is a fixed point on mapping $T$. Thus, we obtain the following expression:

$$
\begin{aligned}
\theta & =\|T v\|=\max _{x \in[0,1]} \int_{0}^{1} G(x, t) \varphi(x, v) \mathrm{d} t \\
& <\frac{\psi(0) n+4 \mu+\left(\alpha^{2}-\alpha\right) q}{\psi(0) \Gamma(\alpha)} \cdot \frac{\psi(0) \Gamma(\alpha)}{\psi(0) n+4 \mu+\left(\alpha^{2}-\alpha\right) q} \cdot\|v\|=\theta .
\end{aligned}
$$

This expression contradicts the hypothesis.

Suppose that boundary problem (3) has a positive solution $v$, and $\|v\|=\theta$, then, $v$ is a fixed point on mapping $T$. Thus, from (iii), we achieve the following:

$$
\begin{gathered}
\theta=\|T v\|=\max _{x \in[0,1]} \int_{0}^{1} G(x, t) \varphi(x, v) \mathrm{d} t \\
>\frac{\alpha^{2}-\alpha}{\psi(0) \Gamma(\alpha)} \cdot \frac{\psi(0) \Gamma(\alpha)}{\alpha^{2}-\alpha}\|v\|=\theta .
\end{gathered}
$$

This expression contradicts the hypothesis.

The proof of (iv) is similar to that of (iii). Hence, such proof is omitted here. In summary, Theorem 3 is proved.

\section{EXAMPLES}

Example 1 Consider the following equation

$$
\left\{\begin{array}{c}
{ }^{C} D^{\frac{9}{2}} v(x)+v^{\beta}(x)+v^{\gamma}(x)=0, \\
0<\beta<1<\gamma, 0 \leqslant x \leqslant 1, \\
v(0)=v^{\prime}(0)=\cdots=v^{(8)}(0)=v^{(10)}(0)=0, \\
v(1)+v^{\prime}(1)=\frac{1}{2} \int_{0}^{\frac{2}{3}} v(t) \mathrm{d} t .
\end{array}\right.
$$

According to (3), in this equation, $\alpha=\frac{9}{2}, n=9, p=$ $q=1, \mu=\frac{1}{2}$, and $\xi=\frac{2}{3}$. We have

$$
\psi(0)=(n+1) p+n(n+1) q-\mu \xi^{n+1} \approx 99.99,
$$

$$
\begin{aligned}
A & =\max \left\{\frac{n}{\Gamma(\alpha)}+\frac{4 \mu \xi^{\alpha-1}}{\psi(0) \Gamma(\alpha)}, \frac{\left(\alpha^{2}-\alpha\right) q}{\psi(0) \Gamma(\alpha)}\right\} \\
& =\max \left\{\frac{9}{\Gamma(4)}+\frac{4 \cdot \frac{1}{2} \cdot\left(\frac{2}{3}\right)^{\frac{7}{2}}}{99.99 \cdot \Gamma(4)}, \frac{\left(\frac{9}{2}\right)^{2}-\frac{9}{2}}{99.99 \cdot \Gamma(4)}\right\} \approx 1.50,
\end{aligned}
$$

and

$$
\begin{aligned}
\int_{0}^{1} t & (1-t)^{\alpha-2}(2-t) \mathrm{d} t \\
& =\int_{0}^{1} t(1-t)^{\alpha-2} \mathrm{~d} t+\int_{0}^{1} t(1-t)^{\alpha-1} \mathrm{~d} t \\
& =\frac{1}{\alpha-1}-\frac{1}{\alpha+1}=\frac{8}{77}
\end{aligned}
$$

We take $\varphi(x, t)=v^{\beta}(x)+v^{\gamma}(x)$. Then

$$
\lim _{v \rightarrow 0} \frac{\varphi(x, v)}{v}=\infty, \lim _{v \rightarrow \infty} \frac{\varphi(x, v)}{v}=\infty .
$$

Therefore, conditions $\left(\mathrm{C}_{1}\right)$ and $\left(\mathrm{C}_{2}\right)$ are valid. We let $\omega=0$. Then for $0 \leqslant v \leqslant \omega$, it yields

$$
\varphi(x, t) \leqslant \omega^{\beta}+\omega^{\gamma}=2<\bar{A}=\bar{A} \omega .
$$

According to Theorem 1 and Remark 2, boundary value problem (12) has at least two positive solutions $\left(v_{1}\right.$ and $v_{2}$ ), and $0 \leqslant\left\|v_{1}\right\|<1<\left\|v_{2}\right\|$.

Example 2 Consider the following equation

$$
\left\{\begin{aligned}
{ }^{C} D^{\frac{5}{3}} v(x)+v^{\beta}(x)+v^{\gamma}(x)=0 & \\
& 0<\beta<1<\gamma, 0 \leqslant x \leqslant 1, \\
& v(0)=v^{\prime}(0)=\cdots=v^{(7)}(0)=v^{(9)}(0)=0 \\
v(1)+v^{\prime}(1)= & \int_{0}^{1 / 3} v(t) \mathrm{d} t
\end{aligned}\right.
$$

According to (3), in this equation, $\alpha=\frac{5}{3}, n=8, p=$ $q=1, \mu=1$, and $\xi=\frac{1}{3}$. We have

$$
\psi(0)=(n+1) p+n(n+1) q-\mu \xi^{n+1} \approx 81.0,
$$

and

$\lim _{v \rightarrow \infty} \inf _{x \in[0,1]}\left(\frac{\varphi(x, v)}{\|v\|}\right)>\frac{\alpha^{2}-\alpha}{\psi(0) \Gamma(\alpha)}=\frac{\left(\frac{5}{3}\right)^{2}-\frac{5}{3}}{81 \cdot \Gamma(1)} \approx 0.01$.

According to Theorem 3, boundary value problem (13) does not have positive solution.

Acknowledgements: This research is supported by the Natural Science Foundation of Shanghai Institute of Technology, Research Fund Nos. ZQ2018-22, $1021 \mathrm{GN} 203006059$. 


\section{REFERENCES}

1. Oldham KB, Spanier J (1974) The Fractional Calculus, Academic Press, New York.

2. Gaul L, Klein P, Kempffe S (1991) Damping description involving fractional operators. Mech Syst Signal Process 5, 81-88.

3. Miller KS, Ross B (1993) An Introduction to the Fractional Calculus and Fractional Differential Equations, John Wiley, New York.

4. Kilbas AA, Srivastava HM, Trujillo JJ (2006) Theory and Applications of Fractional Differential Equations, North-Holland Mathematics Studies, 204, Elsevier, Amsterdam.

5. Lakshmikantham V (2008) Theory of fractional functional differential equations. Nonlinear Anal 69 3337-3343.

6. Kosmatov N (2009) A singular boundary value problem for nonlinear differential equations of fractional order. J Appl Math Comput 29, 125-135.

7. Zhai CB, Xu L (2014) Properties of positive solutions to a class of four-point boundary value problem of Caputo fractional differential equations with a parameter. Commun Nonlinear Sci Numer Simulat 19, 2820-2827.

8. Shi M (2018) Existence of positive solutions of nonlinear fractional differential equations. J Jishou Univ (Nat Sci Edn) 39, 8-13. [in Chinese]

9. Arqub OA, Maayah B (2019) Fitted fractional reproducing kernel algorithm for the numerical solutions of ABC-fractional Volterra integro-differential equations. Chaos Solitons Fractals 126, 394-402.

10. Sahadevan R, Prakash P (2017) On Lie symmetry analysis and invariant subspace methods of coupled time fractional partial differential equations. Chaos Solitons Fractals 104, 107-120.

11. Alsaedi A, Ahmad B, Alghanmi M (2019) Extremal solutions for generalized Caputo fractional differential equations with Steiltjes-type fractional integroinitial conditions. Appl Math Lett 91, 113-120.

12. Mozyrska D, Torres D, Wyrwas M (2019) Solutions of systems with the Caputo-Fabrizio fractional delta derivative on time scales. Nonlinear Analysis: Hybrid Systems 32, 168-176.

13. Bohner M, Hatipoğlu VF (2019) Dynamic cobweb models with conformable fractional derivatives. Nonlinear Analysis: Hybrid Systems 32, 157-167.

14. Jia JM, Wang WX, Yao JX (2018) The existence of positive solutions for a class of fractional differential equations with integral boundary conditions. $J$ Sichuan Normal Univ (Nat Sci) 41, 197-204. [in Chinese]

15. George A (2009) Fractional Differentiation Inequalities, Springer, Dordrecht.

16. Guo DJ (2015) Nonlinear Functional Analysis, Higher Education Press, Beijing. [in Chinese]

17. He Y (2016) Existence and multiplicity of positive solutions for singular fractional differential equations with integral boundary value conditions. Adv Differ Equ 2016, ID 31. 